\title{
Study to Show Changes in Pulmonary Functions Due To Central Obesity
}

\author{
Sonu Ajmani, Ajay Kumar* \\ Department of Physiology, NSCB Medical College, Jabalpur M.P. India
}

\section{ABSTRACT}

Background: Obesity is an important health problem in the world, but also growing fast in the developing countries like India. Specifically, centralobesityisassociatedwithgreaterhealthriskthanlowerbodyobesity.Obesityismeasured using waist circumference (WC) and body mass index (BMI). The association between body mass index, waist circumference and dynamic pulmonary function test (PFT) varies in different populations and shows controversial results.

Methods and Materials: Present study covered 150 healthy sedentary employees of age group (30-40) years, who worked in an air conditioned environment of a private motor vehicle centers at Jabalpur for a minimum of six years. Their anthropometric measurements- height, weight, waist-hip circumference, chest expansion are measured to calculate body mass index, waist-hip ratio and total body fat\%. Spirometry was performed after informed consent in all these subjects. FEV1, FVC, FEV1/FVC ratio, PEFR, FEF 25-75 was recorded.

Results: When PFT variables of low BMI and overweight BMI group was compared with that of normal BMI group, the result shows inverse relation between BMI, WC and FEV1/FVC which indicates an obstructive airway diseases and it would be worsen in genetically prone high BMI subjects. FEF25-75 varies proportionatelywith increasing BMI indicates deterioratinglungfunctions.

Conclusions: An attempt was done to bring awareness about variation of lung functions with increase in BMI and WC. Life style modifications and interventions to keep BMI and WC at normal level might help us in moving forward for eradication of obesity and impairment of pulmonary functions.

Keywords: Pulmonary Function Tests, Central Obesity, Waist Circumference, Body Mass Index

\section{Introduction}

Obesity is a global health hazard and has been linked to numerous metabolic complications such as dyslipidemia, type II diabetes, \& cardio vascular diseases and is negatively associated to the pulmonary function ${ }^{1}$. Prevalence of obesity has become an important health problem in developing countries particularly in India. Central obesity is an important predictor of adverse health effects such as diabetes, hypertension, hyperlipidemia, and coronary events ${ }^{2}$. Abdominal obesity is associated with greater health risk than lower body obesity ${ }^{3}$. It is usually linked with abnormal eating behavior and sedentary lifestyle. Obesity is measured using waist circumference (WC) and body mass index (BMI). Body weight and BMI can be easily measured and therefore are frequently used in large-scale epidemiologic studies to find out the health hazards caused by obesity. Numerous studies have examined the association between body mass index (BMI; in $\mathrm{kg} / \mathrm{m} 2$ ) or body weight and pulmonary function testing variables but varies in different populations $s^{4,5,6}$.
Several studies have also evaluated the relation of waist circumference (WC) and waist-to-hip ratio (WHR) to pulmonary function testing variables with controversial results $^{7,8,9}$. The mechanism which influences lung function in obesity is still debated and the best marker of central adiposity in relation to pulmonary functions is still not clear.

Globalization and technology dependent life leads to decrease in physical activity and substantial dietary changes especially consumption of fast food are important factors causing central obesity. It is well established that a sedentary lifestyle is associated with an acceleration of age-related physiological deterioration, such that the effects of aging and habitual physical inactivity may be additive. Some of these changes will influence the mechanical properties of the respiratory system. A physically active lifestyle is well established as a central component in the maintenance of good health and disease prevention. Earlier studies have not considered the percentage of body fat, BMI, waist hip ratio influencing the pulmonary function tests. Thus, the purpose of this study was to evaluate the 
association of pulmonary functions with the abdominal obesity in sedentary workers.

\section{Materials and Methods}

This study was conducted in a private motor vehicle centers at Jabalpur in a period of six months. A total of 150 employees (30-40 years) who worked in the air conditioned environment for a minimum of 6 years were included in the present study. The employees in the study group were not regularly doing any type of exercise and no previous history of any respiratory diseases. Institutional Medical Ethical committee approval was obtained and written consent was taken from all participants. Workers with any pre-existing respiratory diseases, with the history of smoking, alcohol, chest wall deformities, Neuromuscular diseases, severe Obesity (BMI $>30)$ and history of Pulmonary Tuberculosis were excluded from this study. The volunteers were asked to avoid beverages like tea, coffee and other stimulants and to report with light breakfast in the forenoon to avoid diurnal variations. They were briefed and familiarized with the procedure and self- demonstration of the required tests. The subjects were divided into three groups based on the Body Mass Index according to the WHO recommendations ${ }^{10}$ for Asian population into normal group (BMI18.5-25), low BMI group $(\mathrm{BMI} \leq 18.5)$ and high BMI (BMI 25-30).

A complete spirogram was performed with Spirolab $^{\mathrm{TM}}$ portable spirometer. The test was carried out in a private and quiet room, in a standing position with the nose clip held in position on the nose. The flowvolume timed graphs were taken out in accordance to the criteria based on the American Thoracic Society. The subject was instructed to take a deep breath until the lungs were full and then blow out through mouth as forcibly and as fast and as long as possible till his maximum capacity, sealing the lips tightly around a clean mouthpiece. Best of the three acceptable curves was selected as the recording. Spirometry parameters for analysis like expiratory volume in 1 second (FEV1) forced Vital Capacity (FVC), FEV1 / FVC Ratio, Peak Expiratory Flow Rate (PEFR), Forced expiratory flow FEF - $25-75 \%$ was recorded.

Anthropometric Measurements: The subjects were enquired for any acute respiratory problem and subjected to anthropometry at the point of entry using the standard procedures and instruments.

Age; was recorded from birthday by calendar to the nearest of year ( $<6$ months and $>6$ months).

Standing Height: was recorded without shoes and with light cloths on a wall mounted measuring tape to the nearest of centimeters $(<5 \mathrm{~mm}$ and $>5 \mathrm{~mm})$.
Weight: was recorded without shoes and with light cloths on a weighing machine with a least count of 100 grams.

Body Mass Index: was calculated by the formula of weight (in $\mathrm{Kg}$ ) and height (in meters). BMI = Weight $(\mathrm{Kg}) /$ (height in $\mathrm{m} 2$ ).

Waist Circumference (WC) Measurement: was done with minimal, adequate clothing (light cloths) with feet 25-30 cm apart and weight equally balanced with a tailor's measuring tape in a plane perpendicular to the long body axis at the level of umbilicus without compression of the skin with nearest to $0.1 \mathrm{~cm}(\mathrm{WC}>/=90 \mathrm{~cm})$ were defined as abdominal obesity using WHO Asia Pacific prospective guidelines

Hip Circumference: (HC) measurement was done with minimal, adequate clothing (light cloths) across the greater trochanter with legs and feet together by a measuring tape without compressing the skin fold.

Waist-hip Ratio; is the ratio of WC and HC was calculated and is the measure of central pattern of fat distribution. $(>0.9$ for male and $>0.8$ for females).

Measurement of Chest Expansion: Chest expansion was measured using a non - stretchable measuring tape at the level of $4^{\text {th }}$ intercoastal space, before and after maximum inspiration.

Body-Fat Percentage Measurement: Body fat can be estimated from the Body mass index (BMI). There is a linear relationship between body-fat percentage (BF \%) and BMI, taking age and gender into account. Based on which following prediction formula has been derived - that showed valid estimates of body fat at all ages, in males and females ${ }^{11,12,13}$. The following formula was used to predict the body-fat percentage is based on current BMI, age, and gender: Adult Body Fat $\%=(1.20$ $\times$ BMI $)+(0.23 \times$ Age $)-(10.8 \times$ gender $)-5.4$ [Gender values for male $=1$, female $=0]$.

Statistical Analysis: Statistical analysis was done by using Student's unpaired ' $t$ ' test. P value was taken as significant at 5 percent confidence level $(\mathrm{p}<0.05)$.

\section{Results}

In the present study, the waist hip ratio, waist circumference, body fat $\%$ was significantly lower $(\mathrm{P}<0.001)$ in the low BMI group and significantly higher $(\mathrm{P}<0.001)$ in the high $\mathrm{BMI}$ group. Chest expansion in high BMI group is significantly less $(\mathrm{P}<0.001)$ compared to that of low BMI group. FEV1/ FVC showed a significant reduction $(\mathrm{P}<0.001)$ in the high BMI group and significant improvement $(\mathrm{P}<0.001)$ in low BMI group. FEF 25-75 is significantly reduced in low BMI group $(\mathrm{P}<0.05)$ when compared to high $\mathrm{BMI}$ group. Further FVC and FEV1 did not show any significant changes. 
Table 1: Waist Hip ratio, FVC (L), FEV1 (L), FEV1/FVC (L), PEFR (L), FEF 25-75(L), BODY FAT \%, Waist Circumference (cm) and Chest expansion (cm) in normal, Low BMI and high BMI subjects.

\begin{tabular}{|c|c|c|c|}
\hline PARAMETERS & NORMAL & LOW BMI & HIGH BMI \\
\hline WAIST HIP RATIO & $0.86 \pm 0.04$ & $0.83 \pm 0.02^{* * *}$ & 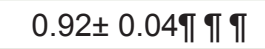 \\
\hline FVC (L) & $3.47 \pm 0.58$ & $3.46 \pm 0.65 \mathrm{NS}$ & $3.46 \pm 0.56 \mathrm{NS}$ \\
\hline FEV1(L) & $3.34 \pm 0.53$ & $3.44 \pm 0.64 \mathrm{NS}$ & $3.30 \pm 0.44 \mathrm{NS}$ \\
\hline FEV1/FVC (L) & $96.54 \pm 4.03$ & $99.26 \pm 1.51^{* * *}$ & 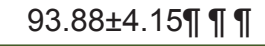 \\
\hline PEFR(L) & $8.94 \pm 1.68$ & $9.05 \pm 1.70 \mathrm{NS}$ & $8.54 \pm 1.13 \mathrm{NS}$ \\
\hline FEF 25-75(L) & $5.09 \pm 1.11$ & $4.73 \pm 1.53^{* *}$ & $5.08 \pm 1.19$ NS\# \\
\hline BODY FAT \% & $16.55 \pm 3.15$ & $9.54 \pm 1.49^{* * *}$ & $22.70 \pm 2.25 \pi$ ा ा \\
\hline Waist Circumference $(\mathrm{cm})$ & $81.18 \pm 7.30$ & $72.45 \pm 6.44^{* * *}$ & $92.15 \pm 5.21 \rrbracket$ ๆ Т \\
\hline Chest expansion (cm) & $3.78 \pm 0.16$ & $4.15 \pm 0.68^{*}$ & $2.36 \pm 0.50$ ा ๆ\# \# \\
\hline
\end{tabular}

( $n=50 ; n$ is the number of subjects in each group).Values expressed as Mean $\pm S D$., $P<0.05^{*}, P<0.001^{* *}, P<0.0001^{* * *}$-Comparison between Normal group versus Low BMI $P<0.001^{\uparrow ~ " ~}, P<0.0001^{\uparrow} \uparrow$ ฯ, - Comparison between Normal group versus High BMI., $P<0.05^{\#}, P<0.001^{\# \#}$ _Comparison between Low BMI versus High BMI.

\section{Discussion}

The health risks related to obesity, including its effects on respiratory function, are not only reflects magnitude of obesity but also due to the presence of abdominal fat ${ }^{14}$. Obesity does not cause airflow obstruction but can result in pulmonary restriction and reduction in airway ${ }^{15}$. Obesity is a condition that may contribute to airway hyper responsiveness ${ }^{15}$.

In the developed and developing countries, due to urbanization of the population in combination with low physical activity, central obesity is increasing. Abdominal obesity is due to accumulation of fat inside the peritoneal cavity, packed between the internal organs. Visceral fat is composed of several adipose depots including mesenteric, epididymal white adipose tissue and perirenal fat. In recent years scientists have come to an important recognition that body fat, instead of body weight, is the key to evaluate obesity. Available literature shows that BMI illustrates the best estimate of the total body fat, while waist measurement gives an estimate of visceral fat and risk of obesity-related disease ${ }^{16.17,18}$. Physical inactivity is considered a global health concern and the leading risk factor for chronic disease and death. In the present study, waisthip ratio, waist circumference and body fat percentage was less in the low BMI group as compared to the high BMI group. Vigorous and high physical activity have stronger relationships with low body fat than moderate activity ${ }^{19}$. The present study showed a significant associations between low physical activity at work and both total and central obesity. These findings provide some support to the hypothesis that low levels of physical activity may be contributing to obesity epidemic. A recent study suggested that it might be possible to increase physical activity at work without sacrificing productivity ${ }^{20}$. Our findings suggest that increasing opportunities for physical activity at work as well as in leisure time and/or reducing long working hours per week should be major components of public health policy for obesity prevention.

Pulmonary function test is a basic and essential test for diagnosis and assessment of pulmonary dysfunction, pulmonary diseases, and treatment effects. Literature shows that distribution of body fat affects pulmonary function $^{21,} 22$. Larger the waist lesser will be the pulmonary function. With an increasing abdominal fat deposition, thoracic volume reduces consequently. Further, not only vital capacity but power of respiratory muscles decreases deteriorating mechanical efficiency of thorax. In addition, pulmonary capacity declines and as a result of shallow and rapid respirations, respiratory dead space increases, and eventually tracheal obstruction and partial atelectasis develop. Previous studies showed an inverse relation between FVC, FEV1 and abdominal obesity $^{23,}{ }^{24}$.Our study did not include obese subjects. Abdominal fat is more likely to have direct effect on the downward movement of the diaphragm, thus reducing the lung volumes and capacities. Central obesity has a clear potential to have a direct effect on respiratory well-being, since it increases oxygen consumption and carbon dioxide production, while at the same time it stiffens the respiratory system and increases the mechanical work needed for breathing. The association between obesity and asthma has also raised new concerns about whether the mechanical effects of obesity on the respiratory system contribute to airway dysfunction that could induce or worsen asthma ${ }^{6}$.A PEFR or 'peak flow' is a measurement of the fastest air flow during forced expiration. When the airways are narrowed by 
bronchoconstriction during an asthma attack, the airflow is reduced, so the PEFR falls. The PEFR may start to fall before the patient is aware of symptoms, and be useful as an early warning of an asthmatic attack ${ }^{25}$. In our study group, the subjects are not showing any significant change in PEFR which indicates that, reduction in FEV1/ FVC in these subjects is not due to developing asthma, but if they are genetically susceptible, an asthma attack would worsen the condition in overweight subjects.

FEF 25-75 showed a marked reduction in low BMI group in our study which could be due to malnutrition. A decrease in the BMI in the underweight population will also lead to a decrement in the pulmonary functions. A reduction in the pulmonary functions might be associated with lack of energy in the underweight population ${ }^{26}$. Malnutrition unfavorably influences the lung functions by decreasing the respiratory muscle mass, strength, endurance and the defense mechanisms of the lung immune system ${ }^{27}$. Muscle wasting leads to reduction in the diaphragmatic mass and a weaker respiratory muscle function diminishes the respiratory muscle strength and it changes the ventilatory capacity of lungs.

\section{Conclusion}

This study is an attempt to bring awareness about variation of lung functions with increase in BMI and WC. The information may help to acknowledge the pulmonary health risks that crop up with increasing body mass index and abdominal fat accumulation. Awareness to maintain BMI and WC at normal levels by life style modifications and interventions might help us in moving forward for eradication of obesity and impairment of pulmonary functions. Moreover the early identification of individuals at risk prior to the onset of obesity is imperative in developing countries. Future research with larger sample size to compare the pulmonary functions with obesity will more enlighten the effect of obesity on pulmonary functions.

\section{References}

1. Saxena Y, Sidhwani G, Upmanyu R. Abdominal obesity and pulmonary functions in young Indian adults: a prospective study. Indian J Physiol Pharmacol. 2009; 53(4):318-26.

2. Raida I. Harik-Khana, Robert A. Wiseb, Jerome L. Flega. The effect of gender on the relationship between body fat distribution and lung function. Journal of Clinical Epidemiology.2001; 54: 399-406

3. Ian Janssen, Peter T Katzmarzyk, and Robert Ross. Waist circumference and not body mass index explains obesity related health risk1-3 Am J Clin Nutr March 2004; 79 (3)379-384.

4. Yue Chen, Donna Rennie, Yvon F Cormier, and James Dosman. Waist circumference is associated with pulmonary function in normal-weight, overweight, and obese subjects. Am J Clin Nutr January 2007; 85(1); 35-39.

5. Heather M. Ochs-Balcom, Brydon J.B. Grant, Paola Muti, Christopher T. Sempos,Jo L. Freudenheim, Maurizio Trevisan, Patricia A. Cassano Licialacoviello, Holger J. Schunemann. Pulmonary Function and Abdominal Adiposity in the General Population. Chest.2006; 129(4):853-862

6. Cheryl M Salome, Gregory G King, Norbert Berend, Physiology of obesity and effects on lung function. J Appl Physiol.2010; 108:206-211.

7. Strachan DP. Ventilatory function, height, and mortality among abdominal obesity and respiratory function. Am J Epidemiol.2004; 159:1140-1149.

8. Carey IM, Cook DG, Strachan DP. The effects of adiposity and weight change on forced expiratory volume decline in a longitudinal study of adults. Int J Obes Relat Metab Disord.1999; 23: 979-985.

9. Chinn DJ, Cotes JE, Reed JW. Longitudinal effects of change in body mass on measurements of ventilatory capacity. Thorax. 1996; 51: 699-704.

10. WHO: Global Database on Body Mass Index. BMI Classification. World Health Organization website.

11. Deurenberg P, Westrate JA, Seidell JC. Body mass index as a measure of body fatness: age- and sex-specific prediction formulas. Br J Nutr.1991; 65(1):105-14.

12. Deurenberg P, Yap M, van Staveren WA. Body mass index and percent body fat.Ameta-analysis among different ethnic groups. Int J Obes Relat Metab Disord.1998; 22(12): 1164-71.

13. Wellens RJ, Roche AF, Khamis HJ, Jackson AS, Pollock ML, Siervogel RM. Relationships between body mass index and body composition. Obes Res. 1996; 4(1): 35- 44.

14. Shinohara, E., Kihara, S. and Yamashits, S. "Visceral fat accumulation as an important risk factor for obstructive sleep apnea syndrome in obese patients", Journal of Internal Medicine.1997; 24124: 11-88.

15. Chlif, M., Keochkerian, D., Mourlhon, C. (2005) "Non invasive assessment of the tension-time index of inspiratory muscles at rest in obese male subjects", International Journal of Obesity. 2005; 29:1478-1483.

16. Rexrode KM, Carey VJ, Hennekens $\mathrm{CH}$, et al. Abdominal adiposity and coronary heart disease in women. JAMA 1998; 280:1843-8.

17. Janssen I, Katzmarzyk PT, Ross R. Body mass index, waist circumference, and health risk: evidence in support of current National Institutes of Health guidelines. Arch Intern Med 2002; 162:2074-9.

18. Chan JM, Rimm EB, Colditz GA, Stampfer MJ, Willett WC. Obesity, fat distribution, and weight gain as risk factors for clinical diabetes in men. Diabetes Care 1994; 17:961-9.

19. Gutin B, Yin Z, Humphries MC, Barbeau P (2005). Relations of moderate and vigorous physical activity to fitness and fatness in adolescents. Am J Clin Nutr 81:746-50. 
20. Healy GN, Dunstan DW, Salmon J, CerinE, Shaw JE, Zimmet PZ, Owen N. Breaks in sedentary time: Beneficial associations with metabolic risk. Diabetes Care .2008; 31:661-666.

21. Ray C, Sue D, Bray G, Hansen J, Wasserman K. Effects of obesity on respiratory function. Am Rev Respir Dis 1983; 128:501-6.

22. Burki NK, Baker RW. Ventilatory regulation in eucapnic morbid obesity Am Rev Respir Dis 1984; 129:538-43.

23. Schunemann HJ, Dorn J, Grant BJ, et al. Pulmonary function is a long-term predictor of mortality in the general population: 29- year follow-up of the Buffalo Health Study. Chest 2000; 118: 656-64.

24. Quanjer PH, Tammeling GJ, Cotes JE, et al. Lung volumes and force ventilatory flows. Report Working Party Standardization of Lung Function Tests, European
Community for Steel and Coal. Official Statement of the European Respiratory Society. Eur Respir J Suppl 1993; 16:5-40.

25. M. Coleman. Teaching Corner: Adult Asthma in Malawi. Malawi Medical Journal. 2011; 23(1): 22-24.

26. UmeshPralhadrao Lad, VilasG. Jaltade, ShitalShisodeLad, P. Satyanarayana. Correlation between Body Mass Index (BMI), Body Fat Percentage and Pulmonary Functions in Underweight, Overweight and Normal Weight Adolescents. Journal of Clinical and Diagnostic Research.2012; 6(3):350-353.

27. Leila Yazdanpanah, FarzadShidfar, Ali JavadMoosavi, Hassan Heidarnazhad, Hamid Haghani. Energy and Protein Intake and Its Relationship with Pulmonary Function in Chronic Obstructive Pulmonary Disease (COPD) Patients. Acta Medica Iranica.2010; 48(6):374-379

*Corresponding author:

Dr Ajay Kumar, Assistant Professor Department of Physiology, NSCB Medical College, Jabalpur M.P. INDIA

Email: acalculia007@gmail.com

Financial or other Competing Interests: None. 